Rev. salud pública. 14 (2): 271-281, 2012

\title{
Evaluación de las prácticas de control de infecciones de los trabajadores de la salud en un hospital de Lima Metropolitana, durante la epidemia de Influenza A H1N1
}

\section{Evaluating healthcare workers' infection control practice in a Lima metropolitan hospital during the influenza $A(H 1 N 1)$ epidemic}

\author{
Martín J. Yagui-Moscosoํㅡㄹ Carolina B. Tarqui-Mamani ${ }^{1}$, Hernán A. Sanabria- \\ Rojas $^{1}$ y Edith E. Encarnación- Gallardo ${ }^{2}$ \\ 1 Instituto Nacional de Salud. Departamento de Medicina Preventiva y Salud Pública de la \\ Universidad Nacional Mayor de San Marcos. Lima Perú. hsanabria@ins.gob.pe \\ 2 Hospital Base María Auxiliadora del Ministerio de Salud. Lima, Perú.
}

Recibido 21 Agosto 2011/Enviado para Modificación 26 Marzo 2012/Aceptado 18 Abril 2012

\section{RESUMEN}

Objetivo Determinar el nivel de cumplimiento de las prácticas de control de infecciones en los trabajadores de la salud en un hospital de Lima Metropolitana durante la epidemia de Influenza A H1N1.

Material y métodos Se realizó un estudio observacional y transversal, se incluyó 165 trabajadores de la salud que brindaban atención a pacientes hospitalizados en áreas de riesgo como servicios de urgencias, emergencia, unidades de cuidados intensivos y hospitalización. El tamaño muestral se calculó a través del programa Epilnfo versión 2000 y se basó en el muestreo sistemático simple. Se utilizó una ficha ad hoc validada por juicio de expertos.

Resultados Del total de encuestados, $23,6 \%$ (39/165) se lavaron las manos antes y después de tener contacto con el paciente, $72,7 \%$ (96/132) utilizaron guantes durante la atención de salud cuando asi estaba indicado, 61,0 \% (64/105) se lavaron las manos después de quitarse los guantes, mientras que 58,0 \% (76/131) de los que tuvieron contacto con material contaminado lo hicieron después de dicho contacto. Solo $14,5 \%(24 / 165)$ de los trabajadores tuvieron prácticas adecuadas. Conclusiones La proporción de prácticas adecuadas de los trabajadores de la salud sobre el control de infecciones durante la epidemia de influenza A H1N1 del 2009 fue baja en el hospital de estudio.

Palabras Clave: Bioseguridad, influenza humana, prevención y control, Perú, epidemiología, servicios hospitalarios (fuente: DeCS, BIREME). 


\section{ABSTRACT}

Objective Determining healthcare workers' level of compliance with infection control practices in a Lima hospital during the influenza $A(\mathrm{H} 1 \mathrm{~N} 1)$ epidemic.

Methods Across-sectional observational study was made of 165 healthcare workers who provided inpatient care in risk areas like emergency services, emergency, intensive care and hospitalisation. The sample size was calculated using Epilnfo software (version 2000) and was based on simple systematic sampling. An ad hoc format validated by experts' judgement was used.

Results $23.6 \%$ (39/165) of the respondents washed their hands before and after contact with patients, $72.7 \%(96 / 132)$ wore gloves for healthcare when so indicated, $61.0 \%(64 / 105)$ washed their hands after removing gloves, while 58.0 $\%(76 / 131)$ of those who had contact with contaminated material did so after such contact. Only $14.5 \%(24 / 165)$ of workers engaged in good practice.

Conclusions The percentage of healthcare workers' engaging in infection control practice in the study hospital during the 2009 influenza A(H1N1) epidemic was low.

Key Words: Biosecurity, prevention, control, Peru, epidemiology, influenza, human, hospital services (source: MeSH, NLM).

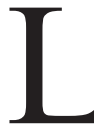

os pacientes con enfermedades infecciosas demandan consultas médicas en todos los niveles de atención, lo que conlleva un riesgo de transmisión para los trabajadores en los establecimientos de salud. El trabajador de la salud que labora en las áreas de riesgo como servicios de urgencias, emergencia, unidades de cuidados intensivos, hospitalización entre otros, pueden infectarse por diversos microorganismos y eventualmente enfermar. El trabajador de la salud pasa de un estado susceptible o expuesto a infectado, potencial enfermo, portador y finalmente, transmisor de microorganismos a otros trabajadores de la salud, pacientes e incluso familiares y miembros de la comunidad.

Uno de los microorganismos patógenos es el virus de la influenza A H1N1; su mecanismo de transmisión es por contacto directo de persona a persona, a través de las secreciones de nariz y boca al toser, estornudar, hablar y cantar sin cubrebocas, cuando el enfermo toca con sus manos a otra persona que ha tosido y estornudado sin lavárselas y también cuando el enfermo besa en la boca a otra persona.

La transmisión puede ser también por contacto indirecto, cuando el enfermo ha tocado objetos con sus manos con secreciones, o tosido y/o estornudado sobre los objetos que no se lavan o limpian y luego otra persona susceptible toca esos objetos y se lleva las manos a la boca, nariz 
y ojos, y lo transfieren a sus respectivas mucosas respiratorias. En este sentido, las medidas para disminuir el riesgo de infección por Influenza (1) son el espaciamiento entre pacientes a más de un metro de distancia, el uso de respiradores convencionales al estar en contacto con el paciente y la protección mediante barreras adicionales como lentes o batas en caso de riesgo de salpicaduras. Se ha documentado la participación de aerosoles en brotes de influenza, especialmente en espacios cerrados (2,3); al respecto las mascarillas quirúrgicas no son efectivas para impedir la inhalación de aerosoles y la superposición de varias mascarillas quirúrgicas no aumenta la eficiencia de protección (4). Se sugiere el uso de los respiradores tipo N95 que tienen una alta eficiencia de filtración y permiten retener partículas de tamaño muy pequeño, como el virus de la influenza, otros agentes virales y el bacilo de Koch.

Existen normas sobre control de infecciones y bioseguridad en los centros asistenciales (5-7); para reducir el riesgo de transmisión de microorganismos vinculados a accidentes por exposición a sangre y fluidos corporales, por ende, reducir las infecciones hospitalarias. El personal de salud debe seguir las precauciones estándar rutinariamente para prevenir la exposición de la piel y de las membranas mucosas; en todas las situaciones que puedan dar origen a accidentes, dichas precauciones deben ser aplicadas a todas las personas, independientemente de presentar o no patologías $(8,9)$.

El 11 de junio del 2009, la Organización Mundial de la Salud declaró la fase 6 de alerta de pandemia de influenza (10), situación que obligó a los trabajadores de la salud a tomar medidas para evitar el riesgo de transmisión de la población en general a ellos y viceversa. En el Perú, el Ministerio de Salud realizó una intensa campaña de difusión acerca de las medidas a adoptar para disminuir el riesgo de contraer la Influenza A H1N1, por lo que era esperable que los trabajadores de la salud hayan maximizado las prácticas de control de infecciones en los nosocomios y establecimientos de salud.

El objetivo del estudio fue determinar el nivel de cumplimiento de las prácticas de control de infecciones en los trabajadores de la salud en un hospital de Lima Metropolitana durante la epidemia de Influenza A H1N1 del año 2009. 


\section{MATERIALES Y MÉTODOS}

Se realizó un estudio observacional y transversal en agosto del 2009 en un hospital ubicado dentro de Lima Metropolitana, Perú. La población de estudio estuvo constituido por los trabajadores de la salud que brindaban atención a pacientes hospitalizados en áreas de potencial riesgo como servicios de emergencia, unidades de cuidados intensivos (UCI), Medicina, Pediatría y Unidades de aislamiento de influenza A H1N1. La recolección de datos se efectuó en un plazo de 10 días para no llamar la atención de los trabajadores.

Ámbito geográfico

La observación se hizo en un hospital público del Ministerio de Salud que brinda atención integral de salud a cerca de dos millones de habitantes, el mismo que corresponde a un establecimiento de referencia para la zona de Lima Metropolitana que cobertura.

Tamaño muestral

Se calculó utilizando el programa EpiInfo versión 2000 bajo los siguientes criterios: 308 camas existentes en los servicios de urgencias, emergencia, unidades de cuidados intensivos, hospitalización y áreas de aislamiento de Influenza AH1N1, nivel de confianza del $95 \%$, precisión del $5 \%$, proporción de trabajadores que se lavan las manos $50 \%$. La unidad de muestreo fue la cama ubicada en las áreas de riesgo. La muestra fue de 171 camas, seleccionadas aleatoriamente a través del muestreo sistemático simple. La unidad de análisis fue el trabajador de salud que brindó atención de salud en las camas seleccionadas, los que fueron 165. Los trabajadores fueron 30,3 \% (50/165) enfermeras, 23,0\% (38/165) médicos residentes, $16,4 \%(27 / 165)$ técnicos de enfermería, 13,9 \% (23/165) médicos asistentes, 3,0 \% (5/165) interno o estudiantes de medicina, 0,6\% (1/165) laboratoristas y otros trabajadores $12,7 \%(21 / 165)$.

Instrumentos

Se utilizó una ficha de observación basada en estándares que establece la OMS, validada a través de juicio de expertos, la misma que incluyó: información general del encuestador, datos de la atención de salud y observaciones de las precauciones estándar: higiene de manos del personal de salud, uso de equipo de protección personal, observación de precauciones para gotitas, observación de la separación y/o aislamiento hospitalario, observación de la limpieza y desinfección de ambientes. Para 
la aplicación del instrumento se dispuso de una guía o instructivo para los observadores, asegurándose el uso de igualdad de criterios.

Recolección de datos

Luego de obtener el total de camas según servicio, se elaboró un croquis de la ruta a seguir y se distribuyó al personal encargado de la observación de las prácticas de los trabajadores de la salud en los servicios; luego se escogió un punto de arranque aleatorio para iniciar la selección de camas a observar. La observación de la cama seleccionada a través del muestreo sistemático duró una hora en promedio; dicha cama debía estar ocupada por un paciente, y en el caso que no hubo, se procedió a reemplazarla por la cama ubicada al lado derecho. Las observaciones diarias se realizaron entre las 8 am y las 4 pm. Los datos de la observación a los trabajadores de la salud que brindaron atención de salud a los pacientes hospitalizados en la cama seleccionada, se pasaron a la ficha de recolección de datos. Finalmente se hizo un control de calidad de cada una de las fichas de recolección de datos en el propio hospital. Se eliminaron 42 fichas porque tuvieron información incompleta, lo que incluía la no consignación del diagnóstico.

Concomitantemente se obtuvo información de 165 familiares de pacientes en relación con la utilización de mascarillas durante la visita.

Análisis de datos

Previo al análisis univariado realizado, se elaboró una base de datos en SPSS versión 15. La evaluación de las prácticas se realizó por cada trabajador, considerando si la atención brindada requería o no el cumplimiento de la práctica de bioseguridad que indica la OMS, posteriormente, basados en el criterio matemático de percentiles, se categorizó el nivel de cumplimiento de las prácticas, considerando como $100 \%$ al total de prácticas aplicables según el tipo de atención que brindó el trabajador de la salud.

Para el análisis del nivel de cumplimiento de las prácticas se consideraron las siguientes categorías:

Nivel inadecuado: si el trabajador de la salud cumplía el 50 \% ó menos de prácticas correctas.

Nivel regular: cumplimiento del 51 a $75 \%$ de prácticas correctas y;

Nivel adecuado: cumplimiento de más del $75 \%$ de las prácticas correctas. 


\section{Consideraciones éticas}

El protocolo fue previamente revisado y aprobado por el Comité de Ética del Instituto Nacional de Salud. Asimismo, se solicitó la autorización respectiva de la Dirección General del Hospital donde se realizó el estudio y se contó con la aprobación de los jefes de cada uno de los servicios donde se llevó a cabo la investigación.

\section{RESULTADOS}

Sólo 23,6 \% se lavaron antes y después de tener contacto con el paciente; $50,9 \%$ trabajadores de la salud no se lavaron antes ni después de tener contacto con el paciente; 7,9 \% se lavaron las manos sólo antes y 17,6 \% sólo después de tener contacto con el paciente.

Se encontró que $72,7 \%$ utilizaron guantes durante la atención de salud, y sólo $61,0 \%$ se lavaron las manos después de quitarse los guantes. También se observó que $58,0 \%$ tuvo contacto con material contaminado y se lavaron las manos después de tener contacto con dicho el material (Tabla 1).

Tabla 1. Distribución de las prácticas del trabajador de la salud en un Hospital de Lima Metropolitana durante la epidemia de Influenza A H1N1

\begin{tabular}{|c|c|c|c|c|}
\hline \multirow{2}{*}{ Prácticas observadas } & \multicolumn{2}{|c|}{ Incorrecta } & \multicolumn{2}{|c|}{ Correcta } \\
\hline & $\mathrm{N}^{\circ}$ & $\%$ & $\mathrm{~N}^{\circ}$ & $\%$ \\
\hline $\begin{array}{l}\text { Se realizó higiene de manos antes y después de tener } \\
\text { contacto con el paciente }\end{array}$ & $84 / 165$ & 50.9 & $39 / 165$ & 23,6 \\
\hline $\begin{array}{l}\text { Realizó higiene de manos después de quitarse los } \\
\text { guantes }\end{array}$ & $41 / 105$ & 39,0 & $64 / 105$ & 61,0 \\
\hline $\begin{array}{l}\text { Realizó higiene de manos después de tener contacto } \\
\text { con material contaminado, se usen guantes o no }\end{array}$ & $55 / 131$ & 42,0 & $76 / 131$ & 58,0 \\
\hline $\begin{array}{l}\text { Realizó higiene de manos antes y después de } \\
\text { colocarse otros EPP }\end{array}$ & $49 / 165$ & 19,7 & $43 / 165$ & 26,1 \\
\hline $\begin{array}{l}\text { Usó guantes al tocar sangre, fluidos, secreciones, } \\
\text { excreciones, mucosas o piel lesionada }\end{array}$ & $36 / 132$ & 27,3 & $96 / 132$ & 72,7 \\
\hline Usó bata en actividades que generan salpicaduras & $46 / 101$ & 45,5 & $55 / 101$ & 54,5 \\
\hline $\begin{array}{l}\text { Usó lentes de protección en actividades que generan } \\
\text { salpicaduras }\end{array}$ & $59 / 82$ & 72,0 & $23 / 82$ & 28,0 \\
\hline $\begin{array}{l}\text { Usó mascarilla en la atención de pacientes con } \\
\text { sospecha de infección respiratoria al tener contacto } \\
\text { cercano }\end{array}$ & $45 / 125$ & 36,0 & $80 / 125$ & 64,0 \\
\hline $\begin{array}{l}\text { Durante el traslado del paciente con sospecha de } \\
\text { infección respiratoria se le colocó mascarilla }\end{array}$ & $10 / 27$ & 37,0 & $17 / 27$ & 63,0 \\
\hline $\begin{array}{l}\text { Usó respirador N95 en procedimientos que generan } \\
\text { aerosoles (intubación, aspiración secreciones, } \\
\text { reanimación, broncospía, nebulización, etc) }\end{array}$ & $51 / 128$ & 39,8 & $77 / 128$ & 60,2 \\
\hline
\end{tabular}


Por otro lado, sólo $26,1 \%$ de los trabajadores se lavaron las manos antes y después de colocarse el EPP mientras que $29,7 \%$ no se lavaron las manos ni antes ni después de dicha práctica; solo $2,4 \%$ se lavaron las manos antes y 10,9 \% después de colocarse el EPP y $30,9 \%$ no aplicaba la práctica.

En relación al uso de equipo de protección personal, 72,7 \% de los trabajadores de la salud usaron guantes al tocar sangre, fluidos, secreciones, excreciones, mucosas o piel lesionada; $54,5 \%$ usaron bata en actividades que generan salpicaduras; finalmente $28,0 \%$ utilizaron lentes de protección en actividades que generan salpicaduras. Asimismo, 64,0 \% de los trabajadores de salud utilizaron mascarilla durante la atención de pacientes con sospecha de infección respiratoria al tener contacto cercano, también se observó que $63,0 \%$ usaron mascarilla durante el traslado del paciente con sospecha de infección respiratoria y $60,2 \%$ de los trabajadores de la salud usaron el respirador N95 en procedimientos que generan aerosoles como la intubación, aspiración de secreciones, reanimación, broncoscopía, nebulización y otros procedimientos.

De otro lado, 60,0 \% (95/165) de los familiares utilizaron mascarilla durante la visita que realizaron a su paciente mientras que un $44,7 \%$ (71/165) de los pacientes con sospecha de infección respiratoria estuvieron en una habitación de aislamiento.

Con respecto al ambiente de hospitalización, se observó que 70,9\% $(117 / 165)$ de las camas de los pacientes atendidos tenían una separación mayor e igual a 1,0 metro de distancia de la cama con respecto al paciente contiguo, 86,7\% (143/165) de las superficies de la habitación de hospitalización del paciente estuvo limpia. Asimismo, 93,3 \% (154/165) tenían lavamanos disponibles en el lugar de atención, sin embargo, solo $76,4 \%(126 / 165)$ tenían insumos disponibles para la higiene de manos en el lugar de atención, esto es por ejemplo, llave del agua clausurada y ausencia de jabón o desinfectantes para el lavado de manos.

El 15,8\% (26/165) del total de diagnósticos registrados de los pacientes evaluados tuvieron Influenza A H1N1 confirmada por el laboratorio, correspondiendo el resto a diversos diagnósticos médicos que podría incluir Influenza A H1N1 no confirmada. 
Finalmente, en relación al cumplimiento global de las prácticas de control de infecciones, se observó que 53,9 \% (89/165) de los trabajadores tuvieron prácticas inadecuadas, $31,5 \%(52 / 165)$ practicas regulares y solamente $14,5 \%(24 / 165)$ prácticas adecuadas, acorde a la clasificación correspondiente de 3 categorías.

\section{DISCUSIÓN}

Preocupa que en plena pandemia de Influenza AH1N1 solamente la cuarta parte de los trabajadores de la salud se lave las manos antes y después mientras que otra cuarta parte se lave las manos solamente antes o solamente después de tener contacto con el paciente. Sin embargo, los resultados observados fueron superiores a lo observado en médicos y enfermeras que laboraban en el servicio de emergencia de un hospital mexicano en donde el lavado de manos entre la atención de pacientes fue 11,2\% (12). Nuestro hallazgo en relación al lavado de manos fue menor a lo observado en internos de medicina de la Universidad Autónoma de Santo Domingo en el Hospital Luis Aybar (13) y lo reportado por Ávila y col en establecimientos de salud ubicados en Puno, Tacna y Piura (14). Al respecto, desde mediados del siglo XIX las manos han sido reconocidas como uno de los principales vehículos (15) para la transmisión de microorganismos, por tanto, el lavado de las manos antes del contacto con cada paciente es considerado como piedra angular de los programas de control de infecciones (16) y la medida más costo-efectiva en reducir la infección hospitalaria. El lavado de manos en nuestro estudio se encuentra en el rango porcentual de la mayoría de los hospitales entre el $16 \%$ y $60 \%$ (17). Las diferencias encontradas podrían explicarse, además, porque la recolección de datos fue por observación directa mientras que en los otros estudios fue a través de un cuestionario.

En relación al uso de los protectores personales, nuestros resultados son similares a lo reportado por Ávila y col. en los servicios de emergencia (14) e inferiores a lo encontrado en internos de medicina de la Universidad de República Dominicana (13). Con respecto al uso de guantes, al tocar sangre, fluidos, secreciones, excreciones, mucosas o piel lesionada, se observó que $72,7 \%$ si utilizan los guantes, resultados ligeramente similares a lo encontrado en trabajadores de la salud del servicio de emergencia de Puno, Tacna y Piura cuya frecuencia fue 89,8 \% (14). 
En relación al nivel de cumplimiento de las medidas de seguridad, es muy preocupante el bajo porcentaje de prácticas adecuadas encontrado; esta situación es paradójica dado que se espera que durante las epidemias de la Influenza A H1N1 se maximicen las medidas de bioseguridad para evitar la infección de los trabajadores de la salud. Se ha señalado razones por las que no se cumplen las prácticas de bioseguridad: actitud de la organización, falta de conciencia por parte de los trabajadores de la salud, barreras logísticas como son los lavamanos lejos de la cama de los pacientes, irritación y xerodermia de la piel producida por los jabones, el exceso de trabajo, una inadecuada percepción del riesgo de los procedimientos realizados de rutina y la falsa sensación de seguridad con el uso de guantes $(18,19)$. Al respecto, hubiera sido valioso averiguar porqué los trabajadores no adoptaban prácticas adecuadas de bioseguridad y así planificar capacitaciones. Rivera y colaboradores en Tacna encontraron que después de una intervención educativa el nivel de prácticas buenas aumentaron de $39 \%$ a $62 \%$ (20). Los resultados son mucho más preocupantes si consideramos que este estudio se hizo cuando el Ministerio de Salud (MINSA) implementó un programa de emergencia para difundir entre la población las medidas preventivas de la influenza A H1N1 y coordinó con diferentes instituciones y sectores para efectuar acciones encaminadas a minimizar el impacto de la epidemia promoviendo comportamientos saludables en la población en general y el personal de salud a través de los medios de comunicación masiva (televisión, radio, vehículos de transporte urbano, periódicos e incluso dentro del portal web oficial (21); además, el hospital donde se llevó a cabo la investigación es uno de los hospitales centinela para la vigilancia de la influenza en general desde inicios de la década (22).

El estudio corrobora lo señalado por el Comité de Revisión de la OMS sobre funcionamiento de las regulaciones de salud internacional (2005) y de la pandemia de influenza A (H1N1) del año 2009 en su reporte borrador del 28 de marzo del 2011: “...el mundo no está preparado para responder a una pandemia de influenza grave o una emergencia de salud". En este sentido, hay necesidad de capacitar al personal de salud en aspectos de bioseguridad hospitalaria para aumentar su nivel de prácticas adecuadas para enfrentar situaciones de brotes de influenza y otras emergencias similares. Con mayor razón si se ha señalado al personal de salud en contacto con pacientes con influenza A H1N1 como factor de riesgo para adquirir influenza (23), diferente a lo que sucede con la influenza aviar H5N1 (24). Al respecto, la 
guía para el manejo de pacientes con la nueva Influenza A H1N1 publicada por la Sociedad Peruana de Enfermedades Infecciosas y Tropicales y resumida por profesionales del Instituto Nacional de Salud (25), omitió considerar la población de trabajadores de la salud como población en riesgo de contraer la influenza $\mathrm{A} H 1 \mathrm{~N} 1$.

En conclusión, la proporción de prácticas adecuadas de los trabajadores de la salud sobre el control de infecciones durante la epidemia de influenza A H1N1 del 2009 fue baja en el hospital de estudio. Se recomienda iniciar o continuar programas de capacitación periódica en el tema de bioseguridad hospitalaria.

Una de las limitaciones del estudio fue realizar la observación horacama-paciente, sin una selección probabilística de los trabajadores de la salud. Sin embargo, el hecho de seleccionar la cama del paciente para la observación evitó alertar a los trabajadores de la salud para modificar sus prácticas. En realidad no fue sencillo observar las prácticas de control de infecciones en los trabajadores de la salud y que los observadores pasen inadvertidos. Estando los observadores con vestimenta blanca de forma similar a los trabajadores del hospital, evitó llamar la atención de los trabajadores de la salud, de los pacientes y familiares visitantes; creemos que la estrategia permitió captar mejor la realidad sobre el nivel de cumplimiento de las prácticas de control de infecciones por parte de los trabajadores de la salud durante la epidemia

\section{REFERENCIAS}

1. Fica A, Cifuentes M, Ajenjo C, Delpiano L, Febre N, Medina W, Parada Y. Precauciones en la atención de pacientes hospitalizados por influenza aviar H5N1. Rev. chil. infectol. 2006; 23 (4): 290-296.

2. Buxton BC, Kuehnert MJ, Hall CB. Transmission of influenza: implications for control in health care settings. Clin Infect Dis 2003; 37: 1094-101.

3. Moser MR, Bender TR, Margolis HS, Noble G R, Kendal AP, Ritter DG. An outbreak of influenza aboard a commercial airline. Am J Epidemiol 1979; 110: 1-6.

4. Derrick JL, Gomersall CD. Protecting healthcare staff from severe acute respiratory syndrome: filtration capacity of multiple surgical masks. J Hosp Infect. 2005; 59: 365-8.

5. Organización Mundial de la salud. Manual de Bioseguridad en el laboratorio. 2da ed.Washington DC:OMS;1992. pp.5-18.

6. Ponce de León R. Manual de prevención y control de infecciones hospitalarias. Washington DC:OPS; 1995. pp.3-15.

7. CONACYT. Manual de bioseguridad. 2da ed. Subcomité de Bioseguridad. Chile: CONACYT; 1996. pp.5-15. 
8. Malagón-Londoño GM, Pontón L. Administración Hospitalaria. Primera edición. Buenos Aires: Editorial Médica Panamericana; 1998.

9. Manual de Bioseguridad y Salud Ocupacional Hospitalaria. Programa Central de Servicios Especiales. Lima: IPSS; 1997.

10. Organización Mundial de la Salud: El nivel de alerta de pandemia de gripe se eleva de la fase 5 a la fase 6. OMS; 2009.

11. Arevalo H, Cruz R, Palomino F, Fernández F, Guzmán E, Melgar R. Aplicación de un programa de control de infecciones intrahospitalarias en establecimientos de salud de la región San Martín, Perú. Rev. perú. med. exp. salud publica. 2003; 20(2):84-91.

12. Peña-Viveros R, Rodríguez-Moctezuma J, López-Carmona J, Martínez-Hernández $M$, Naranjo-Delgado O. Conocimientos y conductas del personal de salud sobre el lavado de manos en un servicio. Rev. Mex Med Urge. 2002 Abril-Junio: 1(2): 43-47.

13. D 'Oleo CP, Matas MB, Cuevas FM. Conocimientos y Prácticas de Bioseguridad que tienen los Médicos Internos de la Universidad Autónoma de Santo Domingo en el Hospital Dr. Luis Eduardo Aybar. Rev. Méd. Dom. 2007; 68(2):166-169.

14. Ávila J, Munayco C, Gomez J, Nunura J, Canahuiri J. Conocimientos y prácticas sobre la nueva Influenza A (H1N1) en trabajadores de salud y pacientes ambulatorios, Perú (Mayo 2009). Rev Peru Med Exp Salud Publica. 2009; 26(3): 328-32.

15. Stewan DB, Williams JG. Bleeding and purging: acure for puerperal fever? J Hospital Infection 1996: 34:691-709.

16. Pittet $\mathrm{D}$. The role of hospital hygiene in the reduction of antibiotic resistance. Bull Acad Natl Med. 2004; 188:1296-80.

17. Larson EL, Eke PI, Laughon BE. Efficacy of alcohol- based hand rinses under frequentuse conditions. Antimicrob Agents Chemother. 1986;30:542-4.

18. Pittet $D$, Boyce JM. Hand hygiene and patient care: pursuing the Semmelweis legacy. Lancet Infectious Diseases 2001;1:9-20.

19. Boyce JM, Kelliher S, Vallande N. Skin irritation and dryness associated with two hand hygiene regimens: soap and water handwashing versus hand antisepsis with an alcoholic hand gel. Infect Control Hosp Epidemiol. 2000;21:442-8.

20. Rivera R, Castillo G, Astete M, Linares V, Huanco D. Eficacia de un programa de capacitación en medidas básicas de prevención de infecciones intrahospitalarias. Rev Peru Med Exp Salud Publica. 2005; 22(2): 88-95.

21. Ministerio de Salud. Perú unidos contra la Influenza. Lima: MINSA; 2009. [Internet] Disponible en: http://www.minsa.gob.pe/portada/Especiales/2009/AH1N1/index.html. Consultado: 14 Agosto 2009

22. Ministerio de Salud. Oficina General de Epidemiología e Instituto Nacional de Salud. Influenza (Módulos Técnicos). Lima; 2000.

23. Laguna VA, Recuenco SE, Avila J. Influenza. Módulo Técnico. En: Náquira-Velarde C, Sanabria-Rojas H, Núñez-Melgar R, Seraylan-Ormachea S, Nalvarte-Estrada R (Eds.). Aspectos Epidemiológicos. Ministerio de Salud. Lima; 2000. pp. 14-21.

24. Cerna-Barco J, Loro-Chero L, Sanabria-Rojas H, Flores-Luna M, Laguna-Torres A, Kochel T, et al. (Ed.). Influenza Aviar H5N1 en humanos. Lima: Ministerio de Salud; 2006.

25. Soria J, Solari L, Cabezas C, Ticona E. Guía para el manejo de pacientes con la nueva Influenza por virus A (H1N1). Rev Peru Med Exp Salud Publica. 2009; 26(3): 349-57. 